\title{
Transdermal Penetrating Peptide Conjugated Liposomes as Drug Delivery Carrier Comprising Macromolecules
}

\author{
Hyung Jun Lim ${ }^{1}$, Jinseo Lee ${ }^{2}$, Su In Park ${ }^{3}$, Soo Hyeon Heo ${ }^{4}$, Beom-Jin Lee*5, Moon Sam Shin ${ }^{* 6}$ \\ ${ }^{1,{ }^{* 5}}$ Department of Applied Bio Technology \& College of Pharmacy, Suwon, Ajou Univ., Republic of Korea

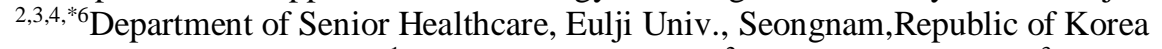 \\ junnumber2@hotmail.com1, dlwlstj97@naver.com²,sooo_30@naver.com ${ }^{3}$, h_soo_h@naver.com ${ }^{4}$, \\ bjl@ajou.ac.kr ${ }^{* 5}$, msshin@eulji.ac.kr ${ }^{* 6}$
}

Article History: Received: 11 January 2021; Accepted: 27 February 2021; Published online: 5 April 2021

\begin{abstract}
The aim of the study was to investigate a system using liposomes and cell penetrating peptides (CPP) for optimal transdermal delivery of macromolecules. Typical DOPE liposomes were prepared with lipid mixture (DOPE:PC:Chol = 1.5:1.5:2.0, molar ratio) and active materials (Rhodamine B; MW 480 and Dextran-RITC; MW 10,000). CPP-conjugated DOPE liposomescontaining active materials were prepared by conjugating the peptide to DOPE liposomes (DOPE:PC:Chol:DSPE-PEG-Mal $=1.5: 1.1: 2.0: 0.2$ or 0.4 , molar ratio). Physical properties of both liposomes were evaluated, including particle size and zeta potential. The particle sizes of typical liposome and CPP-DOPE liposome were approximately $100 \mathrm{~nm}$, and the zeta potential values of both liposomes were approximately $-25 \mathrm{mV}$ and over $+11 \mathrm{mV}$ respectively. Moreover, cellular uptake efficiency was assessed by flow cytometry (FACS). CPP-conjugated liposomes resulted higher cellular uptake efficiency compared to typical DOPE liposomes, showing higher fluorescent intensity in CPP-DOPE liposomes. In confocal laser scanning microscope (CLSM) studies, both cellular uptake and skin permeation were visually estimated. In the case of Rhodamine B, having a relatively small molecular weight,absorption into the cell was successful, and showed the highest rate of cellular uptake with CPP-DOPE liposomes. Dextran-RITC, a macromolecule with a relatively bigger molecular weight, showed similar results to Rhodamine B. In terms of skin permeation, CPP-DOPE liposomes containing Rhodamine B showed noticeable skin absorption after 4 and 18 hours, and the permeation range was wider and thicker than that with typical liposomes. For Dextran-RITC, with typical DOPE liposome, it was hardly permeable through the skin, but with CPP-DOPE liposomes, on the other hand, the skin permeations after 4 and 18 hours were remarkable. The improved cellular uptake and skin permeation of the CPP-conjugated liposomes are due to the cationic arginine-rich peptide. In vivo studies also proved that the CPP-conjugated liposomes are superior in depigmentation and anti-wrinkle studies than typical liposomes. These results demonstrate that the CPP-conjugated liposomes could also be effective for transdermal drug delivery of antioxidant and anti-aging therapeutics.
\end{abstract}

Keywords: Cell penetrating peptides, transdermal delivery, conjugated liposomes, skin permeation.

\section{Introduction}

Generally, hydrophilic and huge substances cannot go into cells due to the barrier of cell membranes. Cell membranes prevent large molecules such as peptides, proteins, and nucleic acids from entering the cell, and even if they were able to enter the cell through a physiological mechanism called endocytosis by cell membrane receptors, they are fused with the cell's resources and eventually decomposed, resulting many restrictions in the treatment and prevention of diseases using macromolecules. Therefore, the development of new strategy that can effectively transmit biomaterials into the body but are not cytotoxic is required and necessary. New alternatives have recently been proposed, of which CPP is under the spotlight as they can increase the availability of large molecules such as siRNA [1-2], protein [3] and peptides [4] that were difficult to utilize as drugs due to low cell membrane permeability and rapid in vivo half-life.

However, using CPP as the transdermal delivery system is still in its infancy.A few studies have reported that CPP such as trans-activatorpenetration [8] can enhance the transdermal delivery of various therapeutic molecules like siRNA, cyclosporine A, insulin, etc. Short oligomers of arginine efficiently cross biological membranes and are more efficient than Tat [9-10] and the third helix of Drosophila antennapedia [11-18]. Short arginine oligomers facilitatetransporting across the cutaneous barrier when applied topically to either mouse or human skin [6]. From the result of these studies, short arginine oligomers are expected to be used as a transdermal delivery system.

Liposomes are widely used drug delivery system in the field of cosmetics and pharmaceuticals because they can contain both hydrophilic and lipophilic components and they can penetrate the intercellular lipids in the stratum corneum. [12]. Due to these advantages, liposomes have been extensively researched for transdermal delivery of active ingredients [13-14].

In this study, we designed a system using liposomes and CPP for optimal transdermal delivery. Physical 
characteristics of the typical liposomes and CPP-conjugated liposomes were investigated. Further, the cellular uptake and skin permeation of both liposomes were examined using flow cytometry and Confocal Laser Scanning Microscope(CLSM), respectively.

\section{Materials and Methods}

\subsection{Materials}

Dioleoylphosphatidylethanolamine (DOPE), phosphatidyl choline (PC), N-[(3-Maleimide-1-oxopropyl) aminopropyl polyethyleneglycol-carbamyl distearoylphosphatidyl-ethanolamine (DSPE-PEG-Mal) were purchased from Avanti Polar Lipids Inc. (Alabaster, AL, USA). Cell penetrating Peptides (GRRRRRRRRRGTLCysteamine) were synthesized by Peptron Co. (Daejeon, Korea). Cholesterol (Chol), cysteine, paraformaldehyde, Rhodamine B, Dextran-Rhodamine B Isothiocyanate (Dextran-RITC),4',6-diamidino-2-phenyl -indole dihydrochloride (DAPI) anddialysis tubingwere purchased from Sigma-Aldrich Co. (St. Louis, MO, USA).Trypsin, bovine serum albumin, and Dulbecco's modified Eagle's medium (DMEM) were purchased from PAA Co. (Pasching, Austria).

\subsection{Preparation of DOPE Liposomes}

DOPE liposomes containing active materials were prepared by employing the thin-film hydration method. Briefly, active materials (Rhodamine B; MW 480 and Dextran-RITC; MW 10,000) and lipid mixture (DOPE:PC:Chol $=1.5: 1.5: 2.0$, molar ratio) were dissolved in $15 \mathrm{~mL}$ chloroform. The solution was then poured into a round-bottom flask, and the solvent was removed by rotary evaporation, forming a thin lipid film on the wall of the flask. Next, $10 \mathrm{~mL}$ distilled water was added to the flask, and the solution was sonicated using a probe-type sonicator (Branson, USA) for 15 min with an amplitude of $40 \%$ and a pulse on/off time of 5 seconds to obtain uniformly sized liposomes.

\subsection{Preparation of CPP-Conjugated DOPE Liposomes}

CPP-conjugated DOPE liposomescontaining active materials (Rhodamine B and Dextran-RITC) were prepared by conjugating the peptide to DOPE liposomes using a thiol-maleimide reaction. Briefly, active materials and lipid mixtures (DOPE:PC:Chol:DSPE-PEG-Mal $=1.5: 1.1: 2.0: 0.2$ or 0.4, molar ratio) were dissolved in $15 \mathrm{~mL}$ chloroform. CPP-conjugated DOPE liposomes were prepared as described in DOPE liposomes preparation with modification. Only distilled water $(8 \mathrm{~mL})$ was added to the flask, because of hydration. The CPP peptide (DSPE-PEG-Mal: peptide $=1.0: 1.1$, molar ratio) was dissolved in $2 \mathrm{~mL}$ distilled water and mixed with the DOPE liposomes. The mixture was stirred with a magnetic stirrer for 12 hours. An excess amount of cysteine was added to block any unreacted maleimide group. The peptides in the solution that were not conjugated to liposomes were removed using dialysis tubing.

\subsection{Particle Size and Zeta Potential}

The particle size and zeta potential of the DOPE liposomes and CPP-DOPE liposomes were measured using an ELSZ analyzer (OtsukaElectronics,Osaka,Japan).TheContinmethodwasusedto resolve particle size distributions. The zeta potentials wereestimated in all cases using the simple Smoluchowski equation. The measurements were repeated three times.

\subsection{Flow Cytometry}

FACS was used to evaluate the ability of cellular uptake of the liposomal systems. Liposomes containing $0.05 \%$ Rhodamine B and 1.0\% Dextran-RITC, Human HaCaT keratinocytes (provided by CLS cell line service) were incubated in DMEM supplemented with $10 \%$ fetal bovine serum (PAA) and $1 \%$ penicillin-streptomycin (PAA) at $37^{\circ} \mathrm{C}$ in a $5 \% \mathrm{CO}_{2}$ atmosphere. $\mathrm{HaCaT}$ cells were pre-incubated in DMEM without fetal bovine serum for $1 \mathrm{~h}$. The cells were incubated of each liposome preparation at $37^{\circ} \mathrm{C}$ for $4 \mathrm{~h}$. Liposomes that did not permeate into the cells were removed by washing with phosphate-buffered saline(PBS) 3 times. The cells were harvested using $0.25 \%$ trypsin, followed by centrifugation and re-suspension in PBS containing $0.1 \%$ bovine serum albumin. The suspensions were embedded in paraformaldehyde ( $3 \%$ in PBS) at $4^{\circ} \mathrm{C}$. The cellular uptake of the fluorescent dye was investigated by using flow cytometry analysis (FACSCalibur, BD).

\subsection{CLSM}

To visualize the skin delivery of DOPE liposomes and CPP-DOPE liposomes, the liposomes were prepared with $0.05 \%$ Rhodamine B and $1.0 \%$ Dextran-RITC. Un-entrapped active materials were removed using dialysis tubing. The skin delivery of DOPE liposomes and CPP-DOPE liposomes were visualizedbyassessingthepenetrationofthe fluorescentdyes by CLSM (LSM510). All optical sections were recorded with the same settings.Rhodamine B was excited at $543 \mathrm{~nm}$ using a $\mathrm{He} / \mathrm{Ne}$ laser, and the fluorescence emission signals were detected at $630 \mathrm{~nm}$ (showninred). 
Table 1: Particle Size andZeta Potential of Liposomal Formulations

\begin{tabular}{|c|c|c|c|c|c|c|c|c|}
\hline Items & $\begin{array}{l}\text { DOPE } \\
\text { liposom } \\
\text { e }\end{array}$ & $\begin{array}{c}\text { CPP } \\
\text { liposome }\end{array}$ & RC & RA4 & RA8 & DRC & DRA4 & DRA8 \\
\hline Lipids & $\begin{array}{l}\text { DOPE:P } \\
\text { C } \\
\text { :Chol }\end{array}$ & $\begin{array}{l}\text { DOPE:PC } \\
\text { :Chol } \\
\text { :DSPE- } \\
\text { PEG-Mal }\end{array}$ & $\begin{array}{l}\text { DOPE:P } \\
\text { C } \\
\text { :Chol }\end{array}$ & $\begin{array}{l}\text { DOPE:PC } \\
\text { :Chol } \\
\text { :DSPE- } \\
\text { PEG-Mal }\end{array}$ & $\begin{array}{l}\text { DOPE:PC } \\
\text { :Chol } \\
\text { :DSPE- } \\
\text { PEG-Mal }\end{array}$ & $\begin{array}{l}\text { DOPE:P } \\
\text { C } \\
\text { :Chol }\end{array}$ & $\begin{array}{l}\text { DOPE:PC } \\
\text { :Chol } \\
\text { :DSPE- } \\
\text { PEG-Mal }\end{array}$ & $\begin{array}{l}\text { DOPE:PC } \\
\text { :Chol } \\
\text { :DSPE- } \\
\text { PEG-Mal }\end{array}$ \\
\hline molar ratio & $\begin{array}{l}1.5: 1.5: 2 \\
.0\end{array}$ & $\begin{array}{l}1.5: 1.1: 2.0 \\
: 0.4\end{array}$ & $\begin{array}{l}1.5: 1.5: 2 . \\
0\end{array}$ & $\begin{array}{l}1.5: 1.1: 2.0 \\
\quad: 0.2\end{array}$ & $\begin{array}{l}1.5: 1.1: 2.0 \\
: 0.4\end{array}$ & $\begin{array}{l}1.5: 1.5: 2 \\
.0\end{array}$ & $\begin{array}{l}1.5: 1.1: 2.0 \\
: 0.2\end{array}$ & $\begin{array}{l}1.5: 1.1: 2.0 \\
: 0.4\end{array}$ \\
\hline $\begin{array}{l}\text { CPP \% per } \\
\text { lipids }\end{array}$ & 0.0 & 8.0 & 0.0 & 4.0 & 8.0 & 0.0 & 4.0 & 8.0 \\
\hline drug (\%) & - & - & $\begin{array}{l}\text { Rhodami } \\
\text { neB }(0.05)\end{array}$ & $\begin{array}{l}\text { Rhodamin } \\
\text { eB }(0.05)\end{array}$ & $\begin{array}{l}\text { Rhodamin } \\
\text { eB }(0.05)\end{array}$ & $\begin{array}{l}\text { Dextran- } \\
\text { RITC (1.0) }\end{array}$ & $\begin{array}{l}\text { Dextran- } \\
\text { RITC (1.0) }\end{array}$ & $\begin{array}{l}\text { Dextran- } \\
\text { RITC (1.0) }\end{array}$ \\
\hline $\begin{array}{l}\text { particle size, } \\
\mathbf{n m}\end{array}$ & $\begin{array}{l}81.34 \pm \\
0.26\end{array}$ & $\begin{array}{l}108.3 \pm \\
0.21\end{array}$ & $\begin{array}{l}117.7 \pm \\
0.29\end{array}$ & $\begin{array}{l}122.6 \pm \\
0.21\end{array}$ & $\begin{array}{l}113.2 \pm \\
0.21\end{array}$ & $\begin{array}{l}127.3 \pm \\
0.27\end{array}$ & $\begin{array}{l}115.5 \pm \\
0.38\end{array}$ & $\begin{array}{l}102.1 \pm \\
0.19\end{array}$ \\
\hline $\begin{array}{l}\text { zeta potential, } \\
\text { mv }\end{array}$ & $\begin{array}{l}-27.5 \pm \\
5.2\end{array}$ & $34.8 \pm 6.3$ & $\begin{array}{l}-19.9 \pm \\
6.6\end{array}$ & $14.7 \pm 9.7$ & $\begin{array}{l}22.1 \pm \\
13.5\end{array}$ & $\begin{array}{l}-22.2 \pm \\
3.8\end{array}$ & $11.8 \pm 8.3$ & $20.6 \pm 9.8$ \\
\hline
\end{tabular}

Dextran-RITCwasexcitedat488nmwithanAr laser, and the fluorescence emission signals were detected at $520 \mathrm{~nm}$ (shown in green). To separate the epidermis and the dermis, DAPI was excited at 408nm with an Ar laser, and the fluorescence emission signals were detected at 460nm (shown in blue).

\section{Results and Discussion}

Table 1 represents physical characteristics of the DOPE liposomes and CPP-DOPE liposomes. The particle sizes of both liposomes were about 100nm. The zeta potential of the DOPE liposome and CPP-DOPE liposome was $-27.5 \mathrm{mV}$ and $+34.8 \mathrm{mV}$, respectively. This change was primarily due to the arginine moieties in the cationic peptide, indicating that DOPE liposomes were successfully conjugated with the CPP peptide on the liposomal surface.

\subsection{Cellular Uptake Efficiency}

Figure 1 shows the cellular uptake efficiency of liposomes using the relative fluorescent intensity. The fluorescent intensity of Rhodamine B and Dextran-RITC in samples treated with the CPP-DOPE liposomes (RA4, RA8; DRA4, DRA8 in Table 1) were higher than that of the DOPE liposomes (RC; DRC in Table 1). These data represented that CPP-conjugated liposomes have higher cellular uptake efficiency than typical DOPE liposomes. This could be due to the presence of the cationic arginine-rich peptide in CPP. This could be due to presence of the cationic arginine-rich peptide in CPP. For Rhodamine B, the zeta potential values of the DOPE and CPP-DOPE liposomes were $-19.9 \mathrm{mV}$ in RC, $+14.7 \mathrm{mV}$ in RA4 and $+22.1 \mathrm{mV}$ in RA8, respectively.

For Dextran-RITC, the zeta potential values were $-22.2 \mathrm{mV}$ in DRC, $+11.8 \mathrm{mV}$ in DRA4 and $+20.6 \mathrm{mV}$ in DRA8, respectively.The CPP-DOPE liposomes could easilyapproach the cell membrane, which consists of negatively charged phosphates and sulfates, through electrostatic interactions with the cationic arginine-rich peptide.Therefore, CPP-DOPE liposomes could induce more endocytosis, leading to increased cellular uptake of Dextran-RITC [15-16].
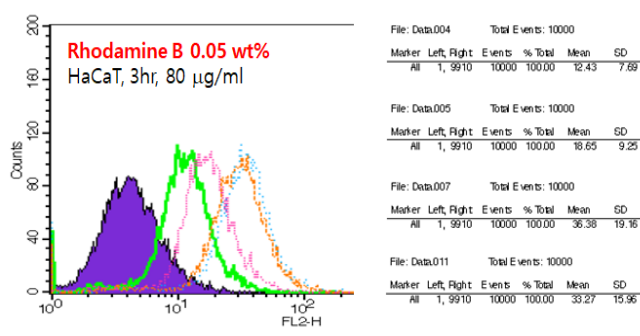

(a) Rhodamine B
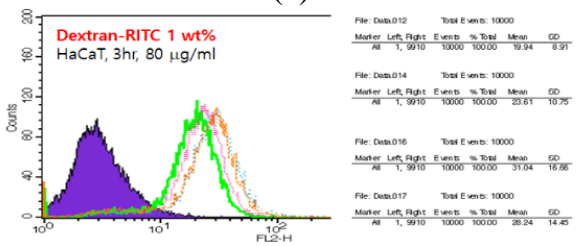

(b) Dextran-RITC
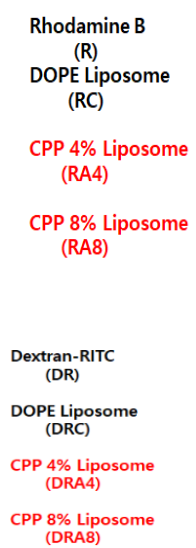
Figure 1:Fluorescence intensity of Rhodamine B and Dextran-Rhodamine B Isothiocyanate (Dextran-RITC) permeated into cells after treatment with DOPE liposomes and CPP-DOPE liposomes containing (a) $0.05 \%$ Rhodamine B and (b) $1.0 \%$ Dextran-RITC.

\subsection{CLSM Study}

Figure 2 shows CLSM images after treating $80 \mu \mathrm{g} / \mathrm{ml}$ of liposomes for 3 hours in the HaCaT cell. Red indicates either Rhodamine B or Dextran-RITC, and blue indicates the nucleus. In the case of Rhodamine B, CPP-DOPE liposome showed the highest rate of cellular uptake, andtypical DOPE liposome showed a little more, compared to the solution in PBS. Dextran-RITC, a macromolecule with a large molecular weight, showed similar results to Rhodamine B. In other words, while PBS solutions or typical DOPE liposomes have little intracellular uptake, CPP liposomes were able to confirm the cellular introduction of a large amount of red fluorescent dyes, which shows that CPP liposome has a significant effect in cellular uptake of not only for small molecules, but also for macromolecules.

Figure 3 presents CLSM images of skin permeation of the DOPE liposomes and CPP-DOPE liposomes containing Rhodamine B and Dextran-RITC.

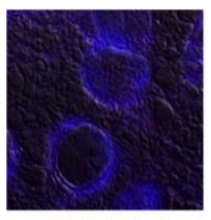

Rhodamine B

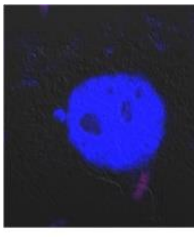

Dextran-RITC

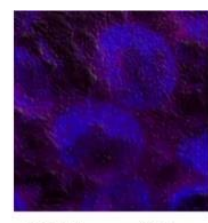

DOPE liposome (RC)

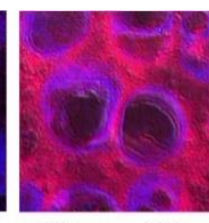

CPP liposome (RA4)

(a) Rhodamine B

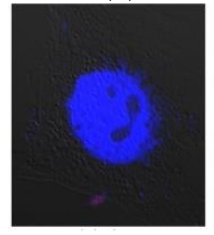

DOPE liposome (DRC)

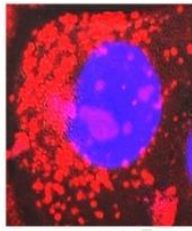

CPP liposome (DRA4)

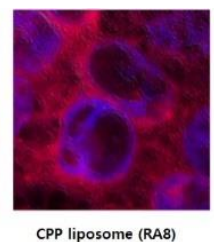

CPP liposome (RA8)

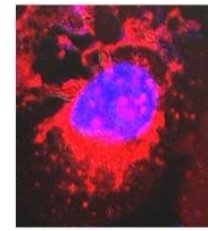

CPP liposome (DRA8)

(b) Dextran-RITC

Figure 2: Confocal laser scanning microscopy (CLSM) images of cell membrane permeation of the DOPE liposomes and CPP-DOPE liposomes containing (a) 0.05\% Rhodamine B and (b) 1.0\% Dextran-Rhodamine B Isothiocyanate (Dextran-RITC).

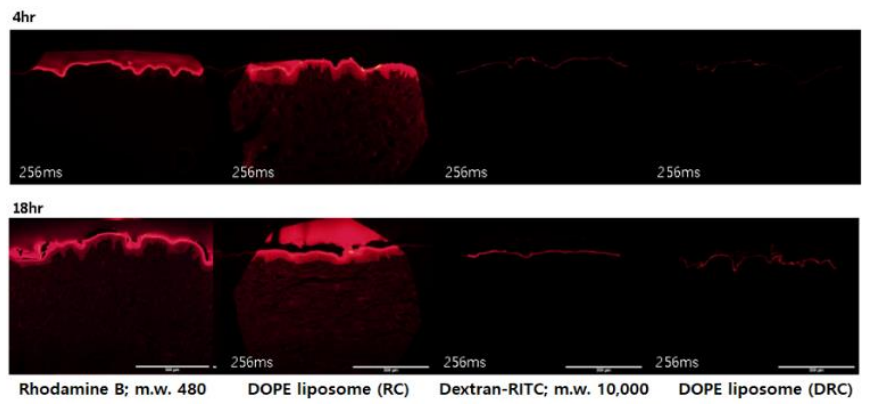

(a) DOPE liposome
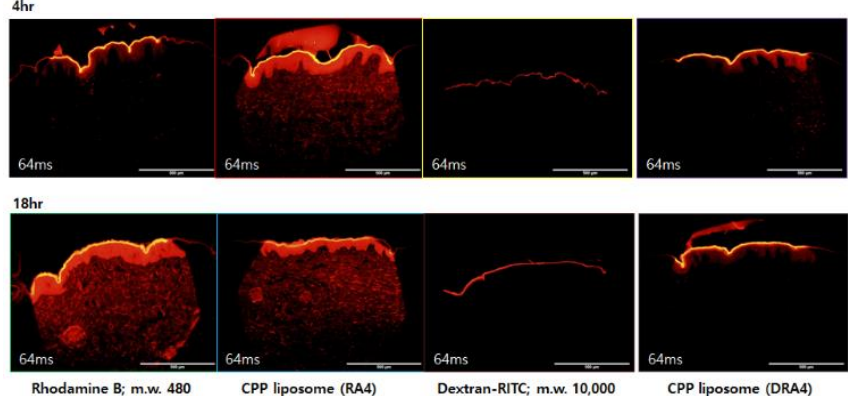

(b) CPP-DOPE liposome

Figure 3:Confocal laser scanning microscopy (CLSM) images of skin permeation of (a) DOPE liposomes and

(b) CPP-DOPE liposomes containing $0.05 \%$ Rhodamine B and $1.0 \%$ Dextran-RITC. 
As shown in Figure 3 (a), in the case of Rhodamine B having a relatively small molecular weight of 480,skin permeation of PBS solution and typical DOPE liposome were checked after 4 and 18 hours. However, in the case of Dextran-RITC having a relatively bigger molecular weight of 10,000, PBS solution and DOPE liposome showed little transdermal permeation after 4 and 18 hours.As shown in Figure 3 (b), CPP-DOPE liposomes containing Rhodamine B showedprominent skin absorption after 4 and 18 hours, and the permeation range was found to be wider and thicker than that with typical DOPE liposome in Figure 3 (a). In the matter of the CPPconjugated liposomes containing high molecular weight Dextran-RITC, the skin permeations after 4 and 18 hours were notably successful, presenting different resultsthan the conventional DOPE liposome shown in Figure 3 (a).

As a result, CPP-conjugated liposomes containing macromolecules, molecular weight about 10,000, were successfully delivered through the cell and the skin by the transdermal drug delivery system. It was because the lipid disturbs the intercellular lipid lamellae of the stratum corneum, and also the positive chargedarginine of the cell penetrating peptide facilitates drug delivery through the electrostatic interaction with negatively charged phosphates and sulfates.Therefore, Rhodamine B and Dextran-RITC encapsulated in the hydrophilic core of the liposomes were able to permeate into deeper skin layers through the stratum corneum.

\section{Conclusions}

We designed the CPP conjugated liposomes as a system using liposomes and CPP for transdermal delivery of macromolecules. The physical characteristics of typical liposomes and CPP-conjugated liposomes containing macromolecules were tested. Further, the cellular uptake of both liposomes were evaluated using flow cytometry. Skin permeation of both liposomes were investigated using Franz diffusion cell and CLSM studies. The particle sizes of both liposomes were approximately $100 \mathrm{~nm}$, and zeta potential values of typical liposomes and CPPconjugated liposomes were approximately $-25 \mathrm{mV}$ and over $+11 \mathrm{mV}$, respectively. Fluorescent-labeled liposomes were prepared to evaluate cellular uptake and skin permeation efficiency. Using flow cytometry, we found that CPP-conjugated liposomes improved cellular uptake of the fluorescent dye as compared with the typical liposomes. In addition, the skin permeation of CPP conjugated liposome was provedhigher than that of typical liposome by CLSM studies. These results were due to the electrostatic interactions between negatively charged phosphates and sulfates, and the cationic arginine-rich peptide. In addition, depigmentation and anti-wrinkle effects of the CPP-conjugated liposomes were proved through in vivo studies. Therefore, it is expected for CPPconjugated liposomes to be applicable for transdermal drug delivery of antioxidant and anti-aging therapeutics as well.

\section{Acknowledgment}

This research was supported bythe Bio \& Medical Technology Development Program of the National Research Foundation (NRF) funded of the Ministry of Science \& ICT (2017M3A9D8048416).

\section{References}

1. Gooding,M., Browne,L.P., Quinteiro,F.M., Selwood,D.L.(2012). siRNA delivery: from lipids to cell-penetrating peptides and their mimics.Chemical Biology \& Drug Design,80,787-809.

2. Nakase,I., Tanaka,G., Futaki,S. (2013). Cell-penetrating peptides (CPPs) as a vector for the delivery of siRNAs into cells.Molecular Biosystems, 9,855-861.

3. Nasrollahi,S.A., Fouladdel,S., Taghibiglou,C., Azizi,E., Farboud,E.S. (2012). A peptide carrier for the delivery of elastin into fibroblast cells.International Journal of Dermatology, 51,923-929.

4. Liu, S., Yang,H., Wan,L., Cheng,J., Lu,X. (2013). Penetratin-mediated delivery enhances the antitumor activity of the cationic antimicrobial peptide magainin II.Cancer Biotherapy and Radiopharmaceuticals, 28,289-297

5. Kamada,H., Okamoto,T., Kawamura,M., Shibata,H., Abe,Y., Ohkawa,A., Nomura,T., Sato,M., Mukai,Y., Sugita,T., Imai,S., Nagano,K., Tsutsumi,Y., Nakagawa,S., Mayumi,T., Tsunoda,S. (2006). Creation of novel cell-penetrating peptides for intracellular drug delivery using systematic phage display technology originated from Tat transduction domain.Biologicaland Pharmaceutical Bulletin, 30, 218-223.

6. Rothbard,J.B., Garlington,S., Lin,Q., Kirschberg,T., Kreider,E., McGrane,P.L., Wende,rP.A., KhavariP.A. (2000). Conjugation of arginine oligomers to cyclosporin A facilitates topical delivery and inhibition of inflammation.Natural Medicine, 6, 1253-1257.

7. Kim,Y.C., Ludovice,P.J., Prausnitz,M.R. (2007). Transdermal delivery enhanced by magainin poreforming peptide.Journal of Controlled Release, 122, 375-383

8. Lee,J., Jung,E., Park,J., Park,D. (2005). Transdermal delivery of interferon-gamma (IFN-gamma) mediated by penetratin, a cellpermeable peptide.Biotechnolgy and Applied Biochemistry, 42, 169173. 
9. Kim,D.T., Mitchell,D.J., Brockstedt,D.G., Fong,L., Nolan,G.P., Fathman,C.G., Engleman,E.G., Rothbard,J.B. (1997). Introduction of soluble proteins into the MHC class I pathway by conjugation to an HIV tat peptide.the Journal of Immunology, 159, 1666-1668.

10. Nagahara,H., Vocero-Akbani,A.M., Snyder,E.L., Ho,A., Latham,D.G., Lissy,N.A., BeckerHapak,M., Ezhevsky,S.A., Dowdy,S.F. (1998). Transduction of full-length TAT fusion proteins into mammalian cells: TAT-p27Kip1 induces cell migration.Nature Medicine, 4, 1449-1452.

11. Derossi,D., Joliot,A.H., Chassaing,G., Prochiantz,A. (1994). The third helix of the Antennapedia homeodomain translocates through biological membranes.Journal of Biological Chemistry, 269,10444-10450.

12. El Maghraby,G.M., Barry,B.W., Williams,A.C. (2008). Liposomes and skin: from drug delivery to model membranes.European Journal of Pharmaceutical Sciences, 34 (4-5), 203-222.

13. Manosroi,A., Kongkaneramit,L., Manosroi,J. (2004). Stability and transdermal absorption of topical amphotericin B liposome formulations. International Journal of Pharmaceutics, 270 (1-2), 279-286.

14. Park,S.N., Lee,M.H., Kim,S.J., Yu,E.R. (2013). Preparation of quercetin and rutin-loaded ceramide liposomes and drug-releasing effect in liposome-in-hydrogel complex system.Biochemical and Biophysical Research Communications, 435, 361-366.

15. Dass,C.R. (2003). Improving anti-angiogenic therapy via selective delivery of cationic liposomes to tumour vasculature. International Journal of Pharmaceutics, 267 (1-2),1-12.

16. Wu,J., Lee, A., Lu,Y., Lee,R.J. (2007). Vascular targeting of doxorubicin using cationic liposomes, International Journal of Pharmaceutics, 337,329-335.

17. Honeywell-Nguyen,P.L., Bouwstra,J.A. (2005). Vesicles as a tool for transdermal and dermal delivery.Drug Discovery Today: Technologies, 2 (1), 67-74.

18. ALTINTAŞ, İ. N., \& KARAASLAN, H. (2019). Peer assessment and active learning experiences of social studies teacher candidates. Review of International Geographical Education Online, 9(2), 319345. 